\title{
Essais
}

ESSAIS

Revue interdisciplinaire d'Humanités

3 | 2013

Narration et lien social

\section{Bonheur individuel et collectif dans le conte philosophique}

\section{Magali Fourgnaud}

\section{(2) OpenEdition}

1 Journals

Édition électronique

URL : https://journals.openedition.org/essais/9155

DOI : $10.4000 /$ essais. 9155

ISSN : 2276-0970

Éditeur

École doctorale Montaigne Humanités

\section{Édition imprimée}

Date de publication : 15 mai 2013

Pagination : 11-31

ISBN : 978-2-9544269-0-7

ISSN : 2417-4211

\section{Référence électronique}

Magali Fourgnaud, «Bonheur individuel et collectif dans le conte philosophique », Essais [En ligne], 3 । 2013, mis en ligne le 01 septembre 2021, consulté le 08 septembre 2021. URL : http://

journals.openedition.org/essais/9155; DOI : https://doi.org/10.4000/essais.9155 


\section{Bonheur individuel et collectif dans le conte philosophique}

\section{Magali Fourgnaud}

Considérer le conte et le mythe comme des ferments du lien social semble une évidence, que l'on songe aux fonctions des mythes fondateurs des civilisations ou aux contes de la tradition orale. C'est d'ailleurs par les histoires que se fait l'entrée dans le langage, si bien que le schéma narratif forge à la fois notre identité et notre perception du réel ${ }^{1}$. C'est pourquoi nous éprouvons la plus grande difficulté à expliquer la fonction du récit et ses enjeux au coeur de la société, comme en témoignent par exemple l'usage et les effets actuels du storytelling ${ }^{2}$. Qu'en est-il du conte littéraire ? Dans quelle mesure peut-il participer au tissage du lien social et à l'émergence d'une conscience politique ?

Au tournant des XVII et XVIII siècles, au moment de ce que Paul Hazard a nommé en 1935 la "crise de la conscience européenne ", un nouveau type de contes apparait. Jacques Barchilon, dans son ouvrage consacré au conte merveilleux de 1690 à $1790^{4}$, souligne en effet l'émergence d'une nouvelle direction du conte de fées dans lequel les personnages parlent " non plus comme des héros de tragédies ou de roman courtois, mais comme de vrais philosophes animés par de nouvelles fictions et participant à une nouvelle préciosité aussi intellectuelle que sentimentale ${ }^{5}$. Dans les discours préfaciels, les auteurs de ce nouveau type de contes, qui fréquentent les salons et les loges maçonniques, revendiquent le rôle de la littérature dans l'avènement d'une " révolution des esprits ", selon l'expression de Voltaire. Dans quelle mesure le conte philosophique participe-t-il au nouveau rapport entre littérature, individu et communauté qui s'établit au début du XVIII' siècle ?

1 Jérôme Bruner, Pourquoi nous racontons-nous des histoires?, Paris, Retz, 2002.

2 Christian Salmon, Storytelling : la machine à fabriquer des histoires et à formater les esprits, Paris, La Découverte, 2007.

3 Paul Hazard, La crise de la conscience européenne : 1680-1715, Paris, France, Gallimard, 1961.

4 Jacques Barchilon, Le Conte merveilleux français de 1690 à 1790, Honoré Champion, Paris, 1975.

5 Ibid., p. 86. 
Cette question s'inscrit dans le cadre d'une réflexion plus générale sur l'articulation entre littérature et opinion publique. Depuis deux décennies, la corrélation entre la littérature et l'émergence de nouveaux modèles de sociabilité au carrefour des XVII et ${ }^{e}$ XVIII ${ }^{e}$ siècle est l'objet d'études aussi bien historiographiques, sociologiques, philosophiques que littéraires. Dans son article Farewell Habermas? Deux décennies d'études sur l'espace public ${ }^{6}$, Stéphane Van Damme a proposé une synthèse de ces recherches et a souligné à la fois la complexité et la fécondité du concept habermassien d'« espace public ». En effet, dans son livre fondateur, L'espace public ${ }^{7}$, Jürgen Habermas a montré comment le développement des échanges de marchandises et d'informations, dans la société pré-capitaliste, a favorisé le passage d'un public considéré comme "sphère publique structurée par la représentation ", à un public compris comme "sphère publique bourgeoise ». Alors que la première se présente comme la reproduction visible des rapports de force, sociaux et politiques, la seconde se définit par le rassemblement d'individus privés (des "capitalistes ", des commerçants, des banquiers, des manufacturiers, mais aussi des médecins, des prêtres, des officiers, des professeurs, des " gens instruits »), critiques à l'égard de la domination politique et économique de l'État, mais également lecteurs. Ces hommes se sont rassemblés en cercles car ils avaient les mêmes préoccupations et les mêmes intérêts, et se sont dotés d'un statut juridique. Ils se considéraient à la fois comme bourgeois, propriétaires de biens et de personnes, et comme hommes (au sens d'êtres humains), et ont puisé dans le modèle familial les principes d'un gouvernement idéal, à savoir, égalité des membres, soin mutuel, recherche de la modération et surtout usage public de sa raison. Ainsi, l'apparition, d'abord en Angleterre, puis en France et en Allemagne, d'une "sphère publique ", constituée de personnes privées qui se réunissent dans des assemblées pour débattre (salons, clubs, loges maçonniques), a été un des facteurs essentiels de la remise en cause de l'absolutisme et de l'émergence d'un nouveau mode de sociabilité fondé sur la raison et l'égalité, et créé sur le modèle des cercles littéraires. Comme Habermas l'a souligné lui-même, il ne s’agit pas de croire que cette idée du public s'est effectivement réalisée à travers les cafés, les Salons et les diverses sociétés ; mais c'est bien grâce à eux qu'elle s’est imposée comme une revendication objective. Il s'agit donc sans doute moins d'une réalité que d'une représentation de ces nouvelles relations sociales, d'une image véhiculée également par la littérature : nous verrons par la suite comment les contes philosophiques

6 Stéphane Van Damme, "Farewell Habermas ? Deux décennies d'études sur l'espace public ", Les Dossiers du GRIHL [en ligne], Les dossiers de Stéphane Van Damme, Historiographie et méthodologie, mis en ligne le 28 juin 2007, consulté le 13 février 2013. [http://dossiersgrihl.revues.org/682;DOI:10;4000/dossiersgrihl.682]

7 Jürgen Habermas, Strukturwandel der Öffentlichkeit, Neuwied, Herman Luchterand Verlag, 1962, L'espace public : archéologie de la publicité comme dimension constitutive de la société bourgeoise, trad. Marc Buhot de Launay, Paris, Payot, 1993, p. 47. Publié en Allemagne en 1962, l’ouvrage a été traduit et édité en France pour la première fois en 1978. 
mettent en scène ces nouveaux modèles de sociabilité et les transmettent. Pour Habermas, la "sphère publique bourgeoise " a été en quelque sorte préfigurée par une "sphère publique littéraire " fondée sur des valeurs morales et rationalistes qui seront les siennes. Ce nouveau public, auquel les biens culturels deviennent accessibles, refuse toute explication ex cathedra et revendique sa capacité à interpréter les œuvres de façon indépendante et rationnelle, à faire usage de son propre entendement. Les questions dont on pouvait discuter deviennent "générales ", au sens où tous devaient pouvoir y prendre part et être capables de porter un jugement. Le principe premier d'égalité des capacités de raisonnement assure la liberté des individus, chacun étant considéré comme "pur et simple être humain":

Les clichés figés en formule de propagande révolutionnaire, l' égalité " et la "liberté" bourgeoises, conservent ici encore un lien vivant avec le contexte dont ils ont surgi : l'usage public que la bourgeoisie (le public bourgeois) faisait du raisonnement seffectuait en principe en faisant abstraction de toute représentation sociale ou politique des hiérarchies, et d'après des règles universelles qui, parce qu'elles restaient rigoureusement extérieures aux individus en tant que tels, leur assuraient un espace où pouvait se développer la dimension proprement littéraire de leur intériorite ${ }^{8}$.

Pour Habermas, l'égalité et la liberté des membres de ces sociétés reposent sur deux principes : l'absence de hiérarchie et l'emploi d'une méthode inductive. Il s'agit en effet de chercher, par l'appel à la raison, un fonds commun universel, au-delà des particularités : «c'est en ce sens que les Physiocrates affirmaient que seule l'opinion publique reconnaissait l'ordre naturel et le rendait sensible, de sorte qu'ensuite le despote éclairé puisse faire de cet ordre naturel, traduit sous la forme de normes générales, la base de sa politique ${ }^{9}$ ». En ce sens, la sphère publique littéraire, qui se caractérise par le développement d'un sentiment d'humanité et par la reconnaissance de l'altérité, acquiert progressivement une efficacité politique.

Mais la force subversive des écrits ne se ressent pas seulement dans la sphère bourgeoise. À la veille de la Révolution, aux côtés de l'élite philosophique et académique, il existe un "prolétariat littéraire ", auteur de pamphlets et de libelles qui sont l'expression et le moyen de diffusion de la colère du peuple à l'égard de la monarchie : ces écrits développent des attaques violentes contre des individus qui détiennent prestige et pouvoir (ministres, courtisans, membres de la famille royale). L'historien américain Robert Darnton a étudié ces pratiques de lectures interdites qui conduisent à l'action révolutionnaire. Il existe ainsi deux circuits littéraires complémentaires, les Lumières, qui minent la confiance de l'élite envers la légitimité de l'ordre établi, et les libelles, qui, en attaquant l'élite, diffusent le mécontentement plus largement et dans des couches plus

8 Ibid., p. 64.

9 Ibid. 
profondes : «Chacun de ces deux camps mérite une place aux origines intellectuelles de la Révolution ${ }^{10}$. » Les libelles et les pamphlets, qui forment la catégorie de livres interdits la mieux vendue au XVIII ${ }^{\mathrm{e}}$ siècle, n'appellent pas à une révolution, ni ne prévoient 1789, ni même ne discutent des transformations politiques et sociales qui permettraient l'abolition de la monarchie : «Sans y penser, elles préparent pourtant cet événement en désacralisant les symboles et en dégonflant les mythes qui avaient fait accepter comme légitime la monarchie à ses sujets ${ }^{11}$ ». Or cette dimension satirique et subversive caractérise également le conte philosophique, lui-même vendu sous le manteau et sous-titré par certains éditeurs de conte " cacouac $^{12}$ ". Le conte philosophique a en outre en commun avec le pamphlet de se présenter comme un récit à clefs, le cadre oriental n'étant que le voile bien transparent de la critique politique.

La diffusion à large échelle de la littérature critique et dénonciatrice a sans nul doute produit une véritable "érosion idéologique ", et transformé la représentation de la monarchie, minant ses mythes fondateurs, tournant en dérision les rituels qui l'exprimaient, habituant les Français à se penser comme les victimes d'un État arbitraire et avili. Mais ne serait-ce pas donner ainsi à la lecture un pouvoir qu'elle n'a pas? Roger Chartier, dans Les origines culturelles de la Révolution ${ }^{13}$ (1990), a rappelé notamment les limites de la circulation de ces livres, l'intérêt éphémère et l'incrédulité des lecteurs, qui ne sont pas forcément convaincus par les libelles et les pamphlets. Il y a donc un risque à lire la littérature " philosophique " à rebours, à partir de l'événement révolutionnaire, et ainsi à lui attribuer une signification univoque, à la fois dénonciatrice et persuasive. En revanche, ce qui aurait rendu pensable, déchiffrable, admissible la rupture avec la monarchie absolue, ce seraient les transformations qui modifient profondément les manières de lire qui se caractérisent par la mobilité du lecteur, l'individualisation du lire, et le désinvestissement religieux de la lecture qui perd sa charge d'autorité et de sacralité :

Avec le triplement ou le quadruplement de la production du livre entre le début du siècle et la décennie 1780, avec la multiplication des institutions qui permettent de lire sans acheter, avec le flux croissant des objets imprimés éphémères (le périodique, le libelle, le pamphlet), une nouvelle manière de lire se répand, qui ôte au livre son statut d'autoritél' .

10 Robert Darnton, Bohème littéraire et révolution, le monde des livres au XVIII siècle, Paris, Gallimard, 1983, p. 33.

11 "Un commerce de livres "sous le manteau" en province à la fin de l'Ancien Régime ", ibid., p. 170.

12 Le terme " cacouac » avait été forgé à partir du grec kakos, méchant, par un auteur anonyme en 1757, pour désigner les Encyclopédistes et les « Philosophes ». " Avertissement du libraire ", $L a$ Reine fantasque, conte cacouac, par M.R.C. de G., 1758 ; voir Anne Defrance et Jean-François Perrin, Contes, Paris, H. Champion, 2008, p. 736.

13 Roger Chartier, Les origines culturelles de la Révolution française, Paris, Éd. du Seuil, 1990.

14 Ibid., p. 114. 
Ainsi, la remise en cause de l'Ancien Régime dépendrait moins de la diffusion des idées que de la désacralisation des idoles par les nouvelles manières de lire et surtout par l'émergence d'un lectorat critique. Roger Chartier souligne également la forte politisation de la sphère littéraire et culturelle. Les associations (clubs, salons, loges) sont des laboratoires où s'invente et s'expérimente, en deçà des discours explicites, une sociabilité " démocratique " qui préfigure celle du jacobinisme : "On peut l'appeler sociabilité démocratique, même si elle n'étend pas son réseau au peuple tout entier, pour exprimer l'idée que les lignes de communication s'en forment "en bas", et horizontalement, au niveau d'une société désagrégée où un homme égale un autre homme, entre les individus de cette société ${ }^{15}$. " Même si ces sociétés semblent respecter le souverain, elles nient, par leur pratique, les fondements de l'ordre traditionnel, hiérarchique et autoritaire. Or le pacte de lecture induit par le conte philosophique instaure également un nouveau rapport entre lecteur et auteur : ce dernier ne se présente pas comme le détenteur d'un sens à transmettre, mais laisse les lecteurs faire " eux-mêmes la moitié ${ }^{16}$ » du chemin, selon l'expression de Voltaire. En effet, fortement symbolique et polysémique, le conte philosophique demande un décryptage et lance ainsi le lecteur dans un processus herméneutique.

Hélène Merlin ${ }^{17}$ a montré, quant à elle, que la notion de public est déjà centrale au XVII ${ }^{e}$ siècle et que l'on ne peut considérer le " public littéraire " du XVIII siècle comme une entité homogène, en rupture avec un "public courtisan et mondain " du XVII siècle. Dès le XVII ${ }^{e}$ siècle, la notion de public suppose un rapport complexe entre le général et le particulier : le public se distingue du particulier, mais il l'englobe. Selon Hélène Merlin, il s'agit moins de l'apparition d'un nouveau public que d'un renversement du rapport entre singulier et collectif au cours du XVII ${ }^{e}$ siècle : ce n'est plus une hiérarchie préétablie qui assure le lien social, c'est désormais la conscience d'avoir en commun une même condition humaine qui unit les hommes ${ }^{18}$. Ce renversement de perspective engage une dimension esthétique. De même que le lien particulier constitue le modèle du lien social, ce ne sont plus ni la terreur ni l'admiration qui constituent le modèle de l'émotion esthétique, mais « l'in-

15 François Furet, Penser la Révolution française, note 1, p. 18, cité par Roger Chartier, ibid., p. 59.

16 "Les livres les plus utiles sont ceux dont les lecteurs font eux-mêmes la moitié ; ils étendent les pensées dont on leur présente le germe ; ils corrigent ce qui leur semble défectueux et fortifient par leurs réflexions ce qui leur parait faible ", Voltaire, Dictionnaire philosophique, éd. Raymond Naves et Olivier Ferret, Paris, Garnier, 2008, p. 4.

17 Hélène Merlin, Public et littérature en France au XVII siècle, Paris, Les Belles Lettres, 1994.

18 "Le public n'est plus, ni sur scène, ni dans la salle, ni dans la vie, une communauté hiérarchiquement ordonnée dont les membres doivent, lors de la représentation théâtrale, éprouver le désir exaltant du dépassement de soi, du sacrifice de leur particulier, mais un collectif de particuliers que la représentation théâtrale fait éprouver tel par le partage mutuel du simple sentiment d'être homme. » Ibid., p. 385 (c'est Hélène Merlin qui souligne). 
térêt individuel d'homme à homme ${ }^{19}$ ", selon l'expression de Marmontel. Comme l'avait montré Habermas, les idées politiques, les concepts juridiques qui serviront à l'élaboration de la république bourgeoise se sont donc bien trouvés éprouvés dans des pratiques " littéraires ", par des pratiques d'écriture, de lecture, de conversations critiques, de sociabilité mondaine. Mais Hélène Merlin souligne le rôle du jeu des représentations elles-mêmes et le type d'émotions, "position de soi, scénographies intérieures, topiques, que ces représentations permettent ${ }^{20}$ ». En ce sens, Hélène Merlin aboutit aux mêmes conclusions qu'Habermas, mais ce dernier privilégie les tendances rationalistes des débats littéraires, sans prendre la mesure de la spécificité de la pratique littéraire et de la puissance esthétique de ses représentations. La littérature ne saurait être réduite à la transmission d'un message, à la représentation d'un représenté. Pour autant, elle favorise bien une forme de communication singulière, non seulement au cœur même du public, mais aussi entre l'auteur et les lecteurs/spectateurs : il s'agit d'une expérience partagée.

Plus récemment encore, l'historienne américaine Lynn Hunt ${ }^{21}$ a montré comment l'expérience culturelle, et pas seulement les échanges d'idées, sont à l'origine des bouleversements individuels qui ont des conséquences collectives. Avant de devenir le fondement des institutions, les "droits de l'homme " désignent un sentiment partagé et véhiculé qui s’appuie sur deux notions : autonomie et empathie. Il s'agit d'être avant tout choqué par tout ce qui désobéit aux règles de la loi naturelle, du droit naturel (premier sens de l'expression « Human rights »). Ce sens du droit naturel demande une autonomie morale et un travail de la raison. Certaines populations en sont exclues, comme les esclaves ou les femmes, considérés comme dépendants économiquement et moralement, de leur propriétaire, de leur père ou de leur mari. Pour Lynn Hunt, la littérature, la peinture et les arts ont joué un rôle majeur dans le développement de cette autonomie morale et de l'empathie ${ }^{22}$. Certes, cette faculté de s'identifier à l'autre et d'imaginer ce qu'il peut ressentir n'est pas née au XVIII' siècle. Elle s'apprend dès le plus jeune âge et se développe dans les interrelations, dans un premier temps, dans un cercle resserré, pour ceux qui sont socialement égaux, les proches, les amis. Selon Lynn Hunt, les lecteurs de romans du XVIII ont développé une nouvelle forme d'empathie qui dépasse les divisions habituelles : nobles-roturiers, maîtres et serviteurs, hommes et femmes, et peut-être même enfants-adultes. Jusque-là, ils acceptaient bien l'égalité des âmes au ciel, mais

19 Jean-François Marmontel, art. "Éloquence poétique », Encyclopédie, cité par Hélène Merlin, ibid., p. 384.

20 Ibid., p. 388.

21 Lynn Avery Hunt, Inventing human rights: a history, New York, États-Unis, Royaume-Uni, W.W. Norton and Company, 2007.

22 "New kinds of reading (and viewing and listening) created new individual experiences (empathy), which in turn made possible new social and political concepts (human rights) ", ibid., p. 33-34. 
ne l'envisageaient pas ici-bas. En revanche, la lecture de romans épistolaires déclenche un processus d'identification aux personnages. Robert Darnton ${ }^{23}$ s'est déjà intéressé aux réactions des lecteurs réels de Rousseau et a souligné la force des larmes et de l'identification aux personnages dans le processus d'éducation morale des lecteurs. L'historien américain a souligné le nouveau mode de communication entre lecteurs et auteurs, communication qui s'apparente davantage à une communion de sentiments : «Auteur et lecteur communient par l'intermédiaire de la page imprimée, chacun d'eux assumant la forme envisagée dans le texte ${ }^{24}$. " Robert Darnton conclut de son étude qu'il n'y a pas de révolution dans les pratiques de lecture à la fin du XVIII siècle, mais qu'une nouvelle façon de lire a conduit les lecteurs à absorber la littérature dans leur vie. Tout en s'inscrivant dans la lignée de Robert Darnton, Lynn Hunt souligne la fonction morale et philosophique de la lecture de romans épistolaires. La fiction donne accès aux lecteurs aux tourments de la conscience de personnages qui leur étaient jusque-là étrangers (valets, femmes...) ${ }^{25}$. Ce partage des émotions et des interrogations morales conduit les lecteurs à se mettre à la place de l'autre : ils s'identifient aux héroïnes, notamment, au point de vouloir non seulement les sauver, mais être comme elles, en dépit de leur destin souvent tragique. C'est qu'ils admirent leur lutte pour l'autonomie, entendue comme lutte contre toute forme de servitude, imposée ou volontaire et cette lutte passe par l'usage que chaque sujet fait de son propre entendement ${ }^{26}$. Penser et décider par soi-même demande des changements psychologiques et politiques, propices à l'élargissement des possibilités de décision individuelle. Or c'est une chose d'expliquer comment la moralité peut être basée sur la raison humaine plutôt que sur l'Évangile et comment l'autonomie est préférable à l'obéissance aveugle ; c'en est une autre de réconcilier cet individu autonome avec le Bien. Une des réponses que donne le XVIII ${ }^{e}$ siècle est l'empathie. Certes, comme le souligne Lynn Hunt, le terme d'empathie est anachronique (il n'apparaît qu'au XXe siècle), mais il définit bien la volonté active de s'identifier aux autres ${ }^{27}$. Il s'agit de permettre au lecteur de développer en lui un "spectateur impartial" qui devient sa boussole morale. Seul un être autonome peut développer ce spectateur, et il ne peut le faire que s'il commence par s'identifier aux autres. Lynn Hunt note que si

23 Robert Darnton, «Le courrier des lecteurs de Rousseau : la construction de la sensibilité romantique ", dans Le massacre des chats, attitudes et croyances dans l'ancienne France, titre original : The Great Cat massacre, traduit de l'américain par Marie-Alyx Revellat, Paris, Robert Laffont, 1985.

24 Ibid., p. 231.

25 " Readers learned to appreciate the emotional intensity of the ordinary and the capacity of people like themselves to create on their own a moral world ", Lynn Hunt, op. cit., p. 58.

26 "If authority justified by divine right, Scripture and history was to be replaced by a contact between autonomous men, then boys had to be taught to think by themselves ", ibid., p. 60 .

27 "The observer is able to become a truly moral being however, only when he takes the next step and understands that he too is the subject of such imaginative identification ", ibid., p. 65. 
l'empathie a joué en faveur des minorités privées de droit, cela n'a pas été le cas pour les femmes: elle a ouvert la voie des droits de l'homme, mais n'en a pas garanti d'emblée l'accès à tous. Les analyses de ces historiens s'appuient sur des romans qui revendiquent leur dimension mimétique et reposent sur l'identification du lecteur au personnage, le roman étant considéré comme un miroir de la vie : comme Robert Darnton, Lynn Hunt étudie la réception de Julie ou la Nouvelle Héloïse de Jean-Jacques Rousseau (1761), et de deux autres romans épistolaires, Pamela (1740) et Clarissa (1747-1748) de Samuel Richardson. L'hypothèse que l'on peut émettre est la suivante : le conte philosophique, qui, au contraire du roman, déréalise les situations et empêche toute projection du lecteur dans le récit, suscite une prise de distance, propice également au développement de "ce spectateur impartial ».

En effet, aux côtés des romans épistolaires, émergent depuis la fin du $\mathrm{XVII}$ siècle une autre forme de récit, qui souligne sa fictionnalité et sa fantaisie, tout en revendiquant également sa capacité à transmettre une vérité sur le plan moral. Il s'agit désormais d'étudier quatre de ces nouveaux contes et de voir comment le texte lui-même met en scène et tisse des relations sociales singulières. Le corpus est constitué de L'Histoire du Prince Titi, de Thémiseul de SaintHyacinthe (1736), Zadig, ou la destinée, histoire orientale, de Voltaire (1748) et Le Palais du silence, conte philosophique, de Philippe de Sainte-Foy d'Arcq (1754). Ces textes soulignent le rapport entre narration et lien social de trois manières : sur le plan de la diégèse, au niveau du dispositif narratif lui-même et surtout par la démarche herméneutique qu'ils suscitent. Dans ces trois approches, l'expression "lien social » est à entendre selon trois acceptions différentes : les membres de la communauté constituée sont unis, soit par les mêmes principes, soit par les mêmes sentiments, soit par la même expérience.

L'Histoire du Prince Titi raconte comment Titi, le jeune prince, juste et éclairé, parvient à prendre le pouvoir grâce à ses qualités et à sa vertu alors qu'il avait été évincé du trône par son cadet, soutenu par le roi et la reine, deux caricatures du pouvoir monarchique. Construit en deux séquences, l'une consacrée à l'accession au pouvoir du prince et l'autre à l'art de gouverner, ce conte a tous les aspects d'un roman de formation, destiné à l'éducation du prince. Zadig, lui aussi, devient, au terme de son parcours, un monarque éclairé, choisi par le peuple pour sa vaillance et sa sagesse : il est le « seul monarque de la terre qui eût un ami », sous le règne duquel Babylone est "gouvernée par la justice et par l'amour ${ }^{28}$ ". Quant au Palais du Silence, il trace également l'accès au pouvoir d'un jeune prince : Iphis, amoureux de Mezronime, la prêtresse de Vénus, s'apprête à renverser son père du trône, avec le soutien de son confident, Protas, lorsque, suite à un tremblement de terre, il se retrouve dans le temple dédié au Dieu du Silence, Harpocrate. Iphis prend alors conscience de ses erreurs grâce aux images projetées sur les parois du sanctuaire : dès qu'il songe à un de ses proches, 
il voit, comme par magie, la scène avec les personnages auxquels il a pensé. Iphis assiste ainsi aux conjurations menées par Protas contre lui, et découvre les manipulations de Mezronime, mais aussi la fidélité de Théone et de son ami Ménocles qui deviendra son " mentor $^{29}$ " une fois qu'il aura recouvré le pouvoir. Comme Zadig, Iphis, une fois de retour chez lui, ramène la paix en Doride qui " calmée par les soins de Clidème [le père d'Iphis] et la mort des séditieux, ne songea qu'au progrès de la vertu, des sciences et des arts ${ }^{30}$. " Par conséquent, les trois contes semblent se présenter tous trois comme des récits d'éducation du prince éclairé, comme des allégories politiques : les histoires illustrent a priori une même conception du gouvernement, à savoir une monarchie éclairée.

Pourtant, les trois auteurs sont loin de partager les mêmes idées. Auteur polygraphe, Thémiseul de Saint-Hyacinthe est considéré comme un fondateur des Lumières ${ }^{31}$, comme un défenseur du despotisme éclairé ${ }^{32}$, voire comme un partisan des Lumières radicales ${ }^{33}$. Il connaît Montesquieu qui travaille alors à l'écriture de L'Esprit des Lois (1748). Loin de délivrer une leçon de politique univoque, le conte de Saint-Hyacinthe est l'occasion d'expérimenter des formes possibles de gouvernement, de mettre à l'épreuve également des théories économiques, au moment-même où cette discipline se développe. Voltaire, bien connu pour sa lutte contre l'" infâme " (la superstition et l'intolérance religieuse) n'en a pas moins alimenté une virulente haine contre Saint-Hyacinthe suite à une échauffourée ${ }^{34}$. Quant au chevalier d'Arcq, descendant direct de Louis XIV ${ }^{35}$, ses textes théoriques (Mes Loisirs ou pensées diverses, suivi de L'Apologie du genre humain, 1755 ; La noblesse militaire ou le patriote français, 1756) montrent sa vive préoccupation pour les difficultés sociales et politiques du pays. Il dédie en 1788-1789 à la ville de Montauban son Invitation à ma patrie en faveur de l'humanité souffrante. Mais les sarcasmes de Grimm dans la Correspondance littéraire et les éloges du Journal de Trévoux, qui salue les œuvres d'un " homme vertueux ", montrent assez les dissensions qui pouvaient exister entre Philippe de SainteFoy d'Arcq et les philosophes réformateurs : " D'Arcq professait des opinions

29 Philippe-Auguste de Sainte-Foy d'Arcq, Le palais du silence. Conte philosophique. Tome premier [-second], Amsterdam [i.e. Paris, Philippe Vincent], 1754, p. 269.

30 Ibid., p. 268.

31 Anic Petzel, Thémiseul de Saint-Hyacinthe (1684-1746) : Studien zum Werk eines Frühaufklärers, Frank-am-Main ; New York, P. Lang, 1994.

32 Élisabeth Carayol, Thémiseul de Saint-Hyacinthe : 1684-1746, Oxford, The Voltaire foundation, Studies on Voltaire and the eighteenth century, $\mathrm{n}^{\circ} 221,1984$.

33 Jonathan Irvine Israel, Les Lumières radicales : la philosophie, Spinoza et la naissance de la modernité, 1650-1750, trad. Pauline Hugues, Charlotte Nordmann et Jérôme Rosanvallon, Paris, Éd. Amsterdam, 2005, p. 647-648.

34 Jean Levesque de Burigny, Lettre... à M. l'abbé Mercier, ... sur les démêlés de M. de Voltaire avec M. de Saint-Hyacinthe..., Londres Paris, s.n., 1780.

35 Jean-Pierre Brancourt, "Un théoricien de la société au XVIII" siècle : le chevalier d'Arcq ", Revue Historique, vol. 250 / 2 (508), octobre 1973, p. 337-362. 
politiques religieuses diamétralement opposées à l'utilitarisme de la secte philosophique $e^{36}$ ", selon Jean-Pierre Brancourt. Il prône en effet un retour à l'austérité et affirme son allégeance à une monarchie absolue dont le principe hiérarchique est l'honneur militaire. Ainsi, si les trois contes philosophiques présentent d'indéniables ressemblances, les trois auteurs sont loin de former une quelconque communauté de pensée. Le conte philosophique n'est pas utilisé comme un outil de propagande ou de diffusion d'une pensée unique : le sous-genre ne cherche pas à cristalliser ou symboliser des référents communs à une même communauté d'individus ; il s'éloigne définitivement de l'allégorie. Pourtant, ces trois ouvrages ont en commun de s'interroger sur les conditions d'une nouvelle sociabilité.

Tout d'abord, quels types de société le conte philosophique peint-il et comment les présente-t-il ? Les trois contes ont en commun d'opposer la société de cour, lieu où règnent l'hypocrisie, les manipulations et l'intérêt personnel, au modèle familial caractérisé comme le lieu de la solidarité, de la quête des biens nécessaires et de la vérité. Dans L'Histoire du Prince Titi, le pouvoir royal est présenté comme une pièce de théâtre ${ }^{37}$ où règnent l'apparat et les faux-semblants et où chacun manie avec adresse l'art de mentir. Le monde artificiel et superficiel de la cour est explicitement mis en opposition avec le mode de vie naturel de la campagne, symbolisé par la famille de Bibi, l'épouse promise de Titi : la sphère publique, lieu de la représentation aristocratique et de l'artificialité, s'oppose ainsi à la sphère privée de la famille bourgeoise. En effet, Abor, le père de Bibi, a lui-même quitté la cour, pour se retirer sur les marges du royaume, après avoir essuyé des perfidies qui ont causé sa ruine ${ }^{38}$. Abor est ainsi présenté comme le modèle du bourgeois, bon père de famille, autonome par son travail et ses possessions : il « travaillait à cultiver son jardin et vivait heureux dans l'innocence avec sa femme et sa fille [qui avaient] assez d'esprit pour être contentes de leur état ${ }^{39}$. " Il est également altruiste car il préfere distribuer son surplus d'argent à " ceux qui n'ont pas leur nécessaire ${ }^{40}$ ». À la différence de la cour, où la défense des intérêts personnels est la cause des conflits, ce qui unit cette communauté, réunie autour d'Abor, c'est avant tout l'amour et l'amitié, considérés comme des vecteurs de clairvoyance et de connaissance ${ }^{41}$.

36 Ibid., p. 340.

37 Thémiseul de Saint-Hyacinthe, La suite de l'histoire du prince Titi, par S. Hyacinthe, dans « Le cabinet des fées; ou Collection choisie des contes des fées et autres contes merveilleux ", Amsterdam / Paris, 1786, t. 28, 1. IV, p. 30-31.

38 Thémiseul de Saint-Hyacinthe, Histoire du Prince Titi, dans « Le cabinet des fées ; ou Collection choisie des contes des fées et autres contes merveilleux ", Amsterdam / Paris, 1786, t. 27, 1. II, p. 393.

39 Ibid.

40 Thémiseul de Saint-Hyacinthe, op. cit., p. 415.

41 "Une âme que l'amour anime en est bien plus habile et plus clairvoyante ; elle trouve en elle un fond de lumière qu'elle n'y aurait pas soupçonné ", ibid., p. 384. 
De même, le portrait, qui ouvre le conte de Voltaire, caractérise Zadig par son humilité et sa modération, son refus " des médisances téméraires, [des] décisions ignorantes ${ }^{42}$ ». Suffisamment riche pour combler ses propres besoins, entouré d'amis, "il crut qu'il pouvait être heureux ${ }^{43}$ ». Pourtant, ce bonheur initial est immédiatement rompu : confronté à la jalousie, à la violence arbitraire et aux superstitions, il déplore sans cesse " qu'il est difficile d'être heureux dans sa vie ${ }^{44}$ ». Mais ce sont justement les épreuves auxquelles il est confronté qui lui permettent de faire usage de son entendement : de raisonnable, il devient sage. À l'image de Salomon, il met à l'épreuve les parties opposées lors des procès, afin de révéler les intentions cachées des deux fils auquel le père veut donner l'héritage, par exemple. Notons que cette démarche (à savoir la quête de la justice par le raisonnement) se répand également dans l'entourage de Zadig : la veuve Almona sauve Zadig du bûcher en dévoilant la concupiscence des prêtres par la ruse. Lors de la scène burlesque du " souper ", au cours duquel une dispute, violente mais absurde, éclate autour des pratiques religieuses, réduisant la foi à des pratiques alimentaires, Zadig parvient à instaurer la paix entre l'Indien, l'Égyptien ou le Grec en soulignant le point commun qui les rapproche : «mes amis, vous alliez vous quereller pour rien car vous êtes tous du même avis ${ }^{45}$ ". Faisant observer à chacun que ce qu'ils adorent, ce n'est ni le gui, ni le bœuf, ni le poisson, mais l'Être qui les a créés, Zadig parvient à unir ainsi toutes les religions, au-delà de leurs différences. On reconnaît certes le point de vue déiste que développera ensuite l'ange Jesrad, mais la démarche inductive, la quête de ce qui est commun, de l'universel au-delà des particularités, parvient à instaurer la tolérance et la paix. Voltaire ne tourne pas en dérision les religions, mais les disputes infructueuses qu'elles déclenchent, lorsqu'on confond le fond et la forme. C'est donc, comme dans L'Histoire du prince Titi, l'amitié et l'usage de la raison, qui unit la société qui entoure Zadig.

Les communautés formées autour de Titi ou de Zadig, unies par un sentiment de fraternité et par une quête de la vérité, annoncent indéniablement la "société des gens de lettres » souhaitée par Diderot et les collaborateurs de L'Encyclopédie. La démarche expérimentale que suivent Titi et Zadig pour apprendre à gouverner en font de véritables princes philosophes : soucieux du bonheur de leurs peuples, préoccupés par la misère dans laquelle vivent leurs sujets, ils s'interrogent sur le meilleur gouvernement possible, gèrent les conflits d'intérêts avec sagesse et discernement. Or cette démarche définit justement la "sociabilité des Lumières ", selon l'expression de Catherine Larrère :

\footnotetext{
42 Voltaire, op. cit., p. 71.

43 Ibid., p. 72.

44 Ibid., p. 79.

45 Ibid., p. 107.
} 
Cette version philosophique de la théorie de la sociabilité élaborée par le droit naturel, et dont d'Alembert fait l'exposé canonique dans le Discours préliminaire de l'Encyclopédie, peut être considérée comme la conception dominante parmi les philosophes. Points communs : la déduction, à partir du besoin individuel ou de l'intérêt personnel, d'une utilité éclairée qui sénonce dans des règles imposant de prendre en compte l'intérêt des autres et capable de venir à bout des passions qui opposent les hommes ${ }^{46}$.

En effet, dans son article consacré à la sociabilité des Lumières ${ }^{47}$, Catherine Larrère montre que la notion de "sociabilité ", au XVIII siècle, n'est pas une manière de présenter les différentes formes de relations sociales, mais d'énoncer une valeur, un principe sur lequel doit reposer la vie en société, à savoir la bienveillance envers les autres. Dans l'article "Sociabilité » de l'Encyclopédie, le Chevalier de Jaucourt définit la notion comme « cette disposition qui nous porte à faire aux hommes tout le bien qui peut dépendre de nous, à concilier notre bonheur avec celui des autres, \& à subordonner toujours notre avantage particulier, à l'avantage commun et général ${ }^{48}$ " et cette disposition vient de Dieu lui-même. De ce principe découlent quatre lois de la société : la quête du bien commun comme règle suprême de toute conduite, l'universalité de l'esprit de sociabilité qui s'étend à toute l'humanité, l'égalité comme un des premiers fondements de la société et le caractère obligatoire de la sociabilité entre les hommes. L'article de l'Encyclopédie s'inscrit ainsi dans la lignée des théories de Grotius et de Pufendorf et affirme le lien entre sociabilité et droit naturel. Les contes philosophiques étudiés témoignent de cette conception de la sociabilité, entendue comme disposition à vivre en bonne société avec ses semblables, à faire usage de sa raison, dans l'intention de canaliser ses passions et de faire un travail sur soi pour dépasser la défense de ses intérêts personnels. Le bonheur individuel ne peut être atteint que s'il est également collectif, idée centrale des Lumières, comme l'a démontré Robert Mauzi dans Lidée de bonheur au XVIII siècle ${ }^{49}$. La fiction joue un rôle essentiel dans ce travail sur soi et dans l'éclairement des consciences.

En effet, ces trois contes ont également en commun de présenter des scénographies de la réception, c'est-à-dire des scènes où sont représentés les retentissements d'un événement chez un sujet non-impliqué : le lecteur, élément tiers, assiste aux effets d'une scène pathétique sur les sentiments et la conscience d'un spectateur. Le conte philosophique met ainsi en scène ses propres effets. Par exemple, au livre VI, Titi rencontre une pauvre paysanne affamée. Le

46 Catherine Larrère, Linvention de l'économie au XVIII' siècle : du droit naturel à la physiocratie, Paris, Presses universitaires de France, 1992, p. 74.

47 Catherine Larrère, article "sociabilité ", dans Dictionnaire européen des Lumières, Paris, Quadrige/PUF, 2007, p. 1147-1150.

48 Le Chevalier de Jaucourt, article "sociabilité ", dans Encyclopédie ou dictionnaire raisonné des sciences, des arts et des métiers, t. 15, p. 251.

49 Robert Mauzi, L’idée du bonheur dans la littérature et la pensée françaises au XVIII siècle, chap. 3 "Le bonheur et ses antinomies ", Genève ; Paris, Slatkine, 1979, p. 140-145. 
champ de vision de Titi, et du lecteur avec lui, est d'abord centré sur l'enfant, abandonné pour un instant par sa mère en train de travailler aux champs ; puis il s'ouvre progressivement et laisse apparaître le personnage féminin. Titi devient spectateur d'une scène de Charité, auquel il est particulièrement sensible : «Elle s'assit sous l'arbre, prit son enfant, et lui donna à sucer une mamelle aride. Le pauvre enfant sourit, et le roi se retourna pour cacher les larmes qui lui vinrent aux yeux ${ }^{50}$. " Le pathétique de la scène suscite la pitié du prince-spectateur et déclenche à sa suite une réaction d'entraide. De même, c'est la rencontre avec le pêcheur qui fait prendre conscience à Zadig de l'existence d'une communauté de malheureux :

L'ardeur de sawver la vie au pêcheur fut aussi prompte que cette réflexion. Il court à lui, il l'arrête, il l'interroge d'un air attendri et consolant. On prétend qu'on en est moins malheureux quand on ne l'est pas seul : mais, selon Zoroastre, ce n'est pas par malignité, c'est par besoin. On se sent alors entraîné vers un infortuné comme vers son semblable. La joie d'un homme heureux serait une insulte; mais deux malheureux sont comme deux arbrisseaux qui, s'appuyant l'un sur l'autre, se fortifient contre l'orage ${ }^{51}$.

Cette scène entre en écho avec celle de la "femme à la mamelle aride " de L'Histoire du prince Titi : même émotion face au sort d'autrui, même empressement à soulager sa douleur. Le jeu des pronoms emporte le lecteur dans le même mouvement empathique : le pronom indéfini unit le personnage, le narrateur et le lecteur avec lui dans un même mouvement de compréhension. On retrouve le même dispositif narratif dans Le Palais du silence : Iphis est médusé par les images qui sont projetées sur les parois du palais. Cette étape de sidération est suivie d'un mouvement de colère contre les traitres, d'empathie à l'égard des victimes et d'amour envers ses fidèles soutiens :

Le péril où Clidème et ma mere demeuraient exposés, la crainte que Menocles n'en fût instruit trop tard, l'impossibilité où j'étais d'agir, la honte d'avoir tant aimé deux personnes si également odieuses et méprisables, le remords d'avoir facilité les devoirs les plus saints, à la passion la plus flétrissante par le choix de son objet, la rage d'avoir été joué si long-tems par des scélérats, l'humiliante découverte de la source de mes égaremens et de mes malheurs dans ma folle vanité, le regret d'avoir méconnu le prix des conseils de Ménocles; enfin tout ce qu'une ame, qui chérit la vertu, peut souffrir après l'avoir trahie, et tout ce que le désespoir peut avoir de tourments, quand la nécessité le désarme, je l'éprouvai, pendant que Mezronime et Protas me developperent les replis ténébreux de leurs caurs ${ }^{52}$.

Certes, les situations qu'Iphis observe ne sont pas étrangères à son sort, mais le lexique de l'émotion associé à celui de la conscience morale exprime l'intériorité du personnage, ce qu'il ressent, immobile face aux différents tableaux qui se

50 Thémiseul de Saint-Hyacinthe, op. cit., p. 252.

51 Voltaire, op. cit., p. 114.

52 Philippe-Auguste de Sainte-Foy d'Arcq, op. cit., t. 2, p. 67-68. 
projettent sur les murs du palais. On assiste bien à une scène pathétique, mais à la différence des romans épistolaires, le pathos est ici médiatisé et mis à distance. En effet, le cadre du conte et la construction du texte en mise en abyme crée un effet de distanciation, comme au théâtre. Le lecteur ne s'identifie pas aux personnages du conte : le lecteur est spectateur des effets des émotions sur la conscience du personnage.

En ce sens, ces trois contes semblent ainsi corroborer l'idée d'une relation étroite entre sensualisme, empirisme, esthétique sentimentale et naissance de l'idée de démocratie, idée qu’a développée David J. Denby ${ }^{53}$. Les scénographies de la réception montrent combien la relation à l'autre se construit à partir du vécu et de la transmission de son expérience : l'observation est suivie immédiatement par un dialogue ou une projection au cours desquelles le spectateur apprend les mésaventures qu'a subies le personnage observé. L'histoire linéaire du héros est ainsi interrompue par un récit imbriqué, qui est une mise en abyme du récit principal. Ce qui est en jeu dans ces scènes, "c'est la représentation d'une sociabilité » :

En réagissant affectivement devant les malheurs d'un autre, le personnage découvre un appel intersubjectif, et l'action qui suit (aumône, consolation, etc.) est un geste social. Le rôle de l'esthétique de l'immobilisation comme un pont entre texte et lecteur permet de compléter l'analyse : le lecteur, implicitement présent comme sujet derrière l'immobilisation, est appelé à se couler dans ces représentations de la sociabilité. [...] d'une représentation de la sociabilité, on est passé à une institution de la sociabilite $e^{4}$.

Le dispositif narratif permet une forte implication du lecteur, non pas par identification aux personnages auxquels il ne ressemble en rien, mais parce qu'il occupe, comme le personnage, une place de spectateur, ému par la scène qu'il observe. Ces scènes de réception sont donc susceptibles de former une communauté d'âmes sensibles, mais également rationnelles. En effet, le personnage n'a pas la fonction de représenter une réalité extérieure à la fiction mais fonctionne plutôt comme une case que chacun peut remplir. Dès lors, le cas singulier prend une valeur universelle : la "femme à la mamelle aride " et le pécheur représentent la misère du peuple, mais également l'humanité tout entière, confrontée aux aléas de la fortune : l'histoire de Zadig devient dès lors celle de la "destinée " humaine. Cette analogie entre l'expérience particulière et l'universel relève d'un processus inductif. C'est en ce sens que l'esthétique sentimentaliste rejoint la science empiriste, mais également la théorie politique :

En politique comme dans l'esthétique sentimentale, la relation entre le cas et la généralité, entre la partie et le tout, est fondée sur une conception scientifique de la représentativité. C'est une manière de saisir la signification, et l'opérativité, de la

53 David J. Denby, "Sensualisme, esthétique sentimentale et démocratie », Dix-huitième siècle, n 31, Paris, Éd. La Découverte, 1999, p. 123-139.

54 Ibid., p. 131. 
notion de droits de l'homme. [...] les hiérarchies, loin d'être données par une autorité quelconque, sont construites par une induction expérimentale : la valeur justice est inséparable des sentiments que je ressens en face des larmes de cet enfant, comme une théorie scientifique vaut dans et par les expériences sur lesquelles elle est bâtie $e^{55}$.

Par conséquent, la dimension philosophique et politique du conte ne tient pas tant au message délivré par une instance narrative, mais plutôt au dispositif narratif lui-même. Placé dans une situation de spectateur d'une scène pathétique, comme le personnage, le lecteur est amené à imaginer et à expérimenter ce que ce dernier ressent. Ainsi, le conte déclenche un mouvement d'empathie, non pas parce que le lecteur se met à la place du personnage, ou s'identifie à lui, mais parce que les émotions du personnage sont universelles et entrent en écho avec celles du lecteur. Ce transfert est possible grâce au rapprochement entre la singularité de l'histoire racontée et son caractère universel. L'universalité des situations met en évidence l'égalité entre personnages, auteur et lecteur : égalité des conditions face à la réversibilité des situations, à la faiblesse humaine, à la mort et égalité des émotions. L'instance textuelle semble ainsi fournir un modèle de substitution, semblable à celui sur lequel repose la démocratie : ce rapport entre singulier et universel et ce principe d'égalité fondent le concept de représentativité. Il ne s'agit pas bien sûr ici de démocratisation des institutions, mais de l'apparition et de la diffusion, avant sa conceptualisation, de l'idée de démocratie, entendue comme refus de toute autorité fondatrice de l'ordre politique (hiérarchie figée, normes imposées) et comme expérience partagée. Comme l'a démontré Jacques Rancière, les relations sociales instituant la démocratie renouent avec une horizontalité qui manifeste une supériorité vide : "une supériorité fondée sur aucun autre principe que l'absence même de supérioritét ${ }^{56}$." Les changements d'angle de vue que permet la lecture des contes philosophiques conduit le lecteur, par l'imagination, à changer de place et de prendre conscience que les places ne sont pas figées. La lecture des contes philosophiques fait ainsi vivre au lecteur une expérience de l'incertitude et de l'indétermination du sens, caractéristique du fait démocratique, selon Claude Lefort. Selon ce dernier, la singularité de la démocratie tient dans une fabrication du sens toujours en suspens. La démocratie fait du lieu du pouvoir un "lieu vide ${ }^{57}$ ", les gouvernants n'étant que des représentants provisoires de ce pouvoir ${ }^{58}$.

Les contes philosophiques placent ainsi les personnages, et le lecteur avec eux, dans un processus de décryptage du sens, aucune autorité ne venant dévoiler de sens caché. L'intersubjectivité favorisée par le conte est ainsi liée non seulement aux scènes de réception, mais également à un processus initiatique. En effet, dans ces trois contes, les trois personnages principaux vivent

55 Ibid., p. 136.

56 Jacques Rancière, La haine de la démocratie, Paris, La Fabrique, 2005, p. 48.

57 Claude Lefort, Essais sur le politique, Paris, Seuil, 1986, p. 28.

58 Fabienne Brugère, Faut-il se révolter?, Paris, Bayard, 2012, p. 60-61. 
une véritable initiation, considérée non seulement comme une formation à l'art de gouverner, mais surtout comme l'accès aux connaissances et à la participation aux mystères de la vie. Ces trois personnages sont contraints de quitter le monde et se retrouvent isolés, Titi sur une île édénique, Zadig au cœur du désert oriental, Iphis au fond d'une grotte puis dans le palais du silence. Ils vivent en ce sens une mort symbolique : en dépassant leur condition première, ils renaissent au monde. Ce changement d'être au monde modifie leur perception et les conduit à porter un nouveau regard sur ce qui les entoure. Le parcours de Zadig peut être lu comme une initiation dans la mesure où il subit une métamorphose entre le début et la fin, dont il récapitule les étapes à chaque nouvelle épreuve : son bonheur initial est lié à sa situation personnelle, en revanche, son bonheur final tient à son acceptation du monde tel qu'il est, à sa reconnaissance des vicissitudes de l'existence. En outre, l'intrigue suit le mouvement d'un progressif obscurcissement suivi d'un mouvement de reconquête : jusqu'à sa " résolution " finale, l'intrigue crôit en complexité, en même temps que s'épaissit le décor oriental, comme si le dispositif narratif symbolisait une abdication momentanée de la conscience devant l'obscurcissement du mystère de la vie, comme l'a montré Jacques Van den Heuvel ${ }^{59}$. Effectivement, dans la première partie du conte, l'atmosphère de Babylone relève plus d'une apparence que d'un réel pittoresque : on reconnaît clairement les réalités contemporaines de l'époque de Voltaire, derrière un décor factice, qui ne sert qu'à voiler la critique politique. En revanche, après son départ de Babylone, Zadig traverse un orient pittoresque marqué par les superstitions et la violence arbitraire, où il se trouve confronté à l'incompréhension et à l'ignorance de ses interlocuteurs. Mais Zadig parvient à retrouver Astarté (principe de lumière, déesse du ciel chez certains peuples sémitiques) et à retourner à Babylone : la boucle ainsi tracée souligne la dimension initiatique du chemin parcouru. En effet, l'apparition de l'ange Jesrad s'apparente à une épiphanie. Zadig est illuminé au sens propre comme au sens figuré par l'ange, " au corps majestueux resplendissant de lumière ${ }^{60}$ ": "les hommes, dit l'ange Jesrad, jugent de tout sans rien connaître : tu étais celui de tous les hommes qui méritait le plus d'être éclairéé ${ }^{61}$ ». Après la révélation, tout se clarifie pour Zadig, le décor s'estompe peu à peu. On se retrouve à Babylone dans un décor aussi stylisé qu'au début.

Dans le conte de Philippe de Sainte-Foy d'Arcq, Iphis suit le même mouvement : une plongée dans le mystère suivie d'une révélation. Larrivée d'Iphis au palais du silence se fait d'abord dans l'obscurité : «ce fut alors, que j'éprouvai

59 Jacques Van den Heuvel, Voltaire dans ses contes : de "Micromégas " à "L'Ingénu », chap. IV "Zadig et le conte et oriental, l'exploitation philosophique des Mille et un », Paris, A. Colin, 1967, p. 196-198.

60 Voltaire, op. cit., p. 133.

61 Ibid. 
tout ce qu'une mort lente, obscure et certaine, peut inspirer d'horreur ${ }^{62}$ ». Les " épaisses ténèbres ", de l' " antre profond " où il se trouve, symbolisent son état mental. En outre, il est réduit au silence : « je sentis que je ne pouvais former aucun $\operatorname{son}^{63}$ ", ce que lui rappelle la statue d'Harpocrate, qui représente " un homme, tenant le doigt sur sa bouche ${ }^{64}{ }^{\prime}$. Il se rend ensuite au palais auquel un " petit escalier de marbre " donne accès :

L'obscurité totale où je me trouvai long-tems, me faisait chanceler à chaque degré que je heurtais : enfin j'arrivai sans accident, jusqu'au faîte de ce pavillon. Une porte basse s'ouvrit d'elle-même, et me rendis la lumière. Je ne sais encore ce qui la produisit. J'entrai dans un cabinet de figure ovale, entièrement revêtu d'acier parfaitement poli, au travers duquel il était impossible qu'aucun astre portât ses rayons. Je me reposai sur une estrade, placée au milieu de ce cabinet ${ }^{65}$.

Le parcours d'Iphis prend ainsi une forte dimension symbolique : son chemin concrétise son cheminement, les progrès de sa conscience et de son discernement. Son parcours se présente ainsi comme une renaissance : "un voile épais venait de se déchirer devant mes yeux : je croyais, pour ainsi dire, penser et sentir pour la première fois ${ }^{66}$ ". À quoi Iphis est-il initié ? À la sagesse. Comme Zadig, il apprend à accepter les faiblesses humaines et accède à un autre niveau de conscience :

Plusieurs épreuves de cette espèce, ne réussirent qu'à mồter l'opinion avantageuse, que j'avais eue de quelques gens célèbres qui se rapetissaient, pour ainsi dire, à l'examen. Satisfait de connaître ceux qui devaient m'approcher, je me promis de ne plus apprendre à mépriser les hommes, en approfondissant leurs vices, leurs faiblesses et leurs erreurs. Je leur tenais compte du soin quils prennent de les voiler: c'est risquer de les haïr, que de vouloir les surprendre avant qu'ils soient préparés à devenir aimables. Je meétais convaincu que l'humanité avait son côté d'imperfection. Je sentis que pour mon plaisir même, il valait mieux le considérer de l'autre ${ }^{67}$.

La conclusion que tire Iphis de son parcours ressemble à s'y méprendre à celle du Prince Titi (le page de Titi, que la fée Diamantine a initié à ses mystères, a significativement comme nom, "l'Éveillé »). En effet, le passage par l'île déserte permet à Titi de prendre conscience que le modèle de gouvernement inspiré du modèle familial et fondé sur « la concorde, les services mutuels et la bonne foi » est utopique ; mais cette désillusion, ce tombeau des rêves, lui permet d'accepter les hommes tels qu'ils sont, comme il l'explique au roi Forteserre, son voisin :

62 Philippe-Auguste de Sainte-Foy d'Arcq, t. 2, op. cit., p. 4.

63 Ibid., p. 11.

64 Ibid., p. 12.

65 Ibid., p. 30.

66 Ibid., p. 95.

67 Ibid., p. 192. 
Nous nous aperçûmes aussitôt que nous étions dans l'erreur, et qu'entre des gens raisonnables, il n'y avait point de gouvernement à établir, puisque l'établissement d'un gouvernement n'est que pour faire faire aux hommes déraisonnables ce que la raison veut qu'ils fassent. [...]Nous passâmes à la considération des hommes tels qu'ils sont, ayant besoin de lois et de magistrats pour remédier à leur extravagance et à leur corruption, et nous prîmes ce royaume et quelques-uns des états voisins pour le plan sur lequel nous devions travailler. Nous parcourûmes les différents arrangements et les divers désordres qui s'y trouvent, et nous cherchâmes avec soin les moyens d'assurer ou de perfectionner les uns, et de remédier aux autres, conformément aux principes de justice pris dans la nature des choses mêmes ${ }^{68}$.

Qualifié de " chimère ", la communauté pacifique d'hommes raisonnables dont rêvent Titi et Bibi est bel et bien un mythe. Pourtant, cet épisode n'en sert pas moins de pierre d'achoppement à la réflexion critique, de déclencheur de la démarche réflexive. Le détour par la fiction, par le mythe, permet paradoxalement de s'ouvrir au monde, de regarder " les hommes tels qu'ils sont ", d'observer « les extravagances ", " les désordres " humains. Cette prise de conscience des faiblesses humaines induit une nouvelle conception des lois civiles : ces dernières n'ont pas une fonction punitive, mais formative ; elles doivent servir de digues aux passions humaines et assurer le vivre-ensemble. On retrouve également ce modèle de gouvernement dans Zadig : "il fit sentir le poids sacré des lois, et ne fit sentir à personne le poids de sa dignitét ${ }^{69}$ ». Par conséquent, le détour par le mythe, par la fiction, prend une dimension initiatique. Il s'agit d'éclairer le prince sur ses propres faiblesses pour qu'il apprenne à se gouverner lui-même avant de gouverner les autres : «l'art de se vaincre soi-même mène à celui de commander aux autres ${ }^{70}$ ", affirme le narrateur du Palais du Silence. Or se connaître soi-même, c'est apprendre à se lire, comme un livre.

En effet, il s'établit, dans ces contes, une communauté de personnages initiés, lecteurs de signes. Si l'initiation a lieu, c'est parce que les trois personnages apprennent à lire les signes qui les entourent : ils ont donc en commun de suivre une démarche herméneutique. Zadig répond brillamment aux énigmes qui lui sont posées, Iphis lit les « chiffres $^{71}$ » que forment les fleurs qui émaillent le gazon. Ce dernier interprète ensuite les images qui se projettent sur les parois du palais du silence comme un livre, et son expérience s'apparente à un véritable voyage en littérature. Il devient spectateur tour à tour de scènes tragiques (la tentative de meurtre de Théone) et burlesques (la dispute entre Xénophane et Pythagore, qui a été lue comme une critique à peine voilée des querelles entre Voltaire et Maupertuis) ; les effets d'ellipses et de sommaire dramatisent l'histoire qui se construit sous ses yeux. Observateur des mœurs,

68 Thémiseul de Saint-Hyacinthe, t. 28, 1. VI, op. cit., p. 260-261.

69 Voltaire, op. cit., p. 86.

70 Philippe-Auguste de Sainte-Foy d'Arcq, t. 2, op. cit., p. 99.

71 Ibid., p. 128. 
Iphis devient lui-même moraliste, faisant le portrait acerbe de l'hypocrite Phérécide. Dès lors, le Palais du silence qui offre "le tableau de l'Univers ${ }^{72}$ " ne pourrait-il pas être lu comme une métaphore de la littérature elle-même ? Titi lui-même lit aussi le monde comme un livre :

Voyez ce papillon, disoit Titi, il est admirable par la beauté des couleurs dont il brille; c'est le symbole de l'inconstance. On doit mépriser les amans qui lui ressemblent. Vous mempêcherez, ma chère Bibi, de lui être jamais comparé; mais, sans vous, j’aurois été papillon. Voyez cette tourterelle, disoit Bibi, c'est le symbole de la fidélité; on dit qu'elle meurt lorsqu'elle perd sa compagne. Je suis tourterelle, mon cher Titi; je serois votre tourterelle, quand vous deviendriez papillon ${ }^{73}$.

Ce passage met en évidence la démarche interprétative et le retour sur soi qu'opère le lecteur : le signe est d'abord observé, puis déchiffré, lu comme symbole d'une attitude morale générale (inconstance ou fidélité), avant d'être transposé à la propre situation du lecteur. L'herméneutique a donc des retombées d'ordre moral, au sens où l'emploi Martha Nussbaum, dans son analyse du roman de James : « les prétentions morales de [c]es textes dépendent de façon centrale de la présence en eux de ce genre de personnages supérieurs, tout à la fois auteurs et interprètes de leur propre vie et dont les lectures de la vie compteront pour nous comme des exemples élevés de la nôtre ${ }^{74}$.»

Les contes philosophiques se caractérisent donc par une écriture fortement symbolique, et non mimétique, ils s'apparentent à des emblèmes, invitant le lecteur à un décryptage. Mais le sens, une fois déchiffré, n’en reste pas moins mystérieux et non univoque. Le narrateur joue en effet avec les différentes interprétations possibles, car lors de la fête organisée en l'honneur de la paix, BibiPsyché est bel et bien transformée en papillon ${ }^{75}$, symbole alors de jalousie. En outre, Titi est comparé non plus à une tourterelle, malgré sa fidélité indéfectible envers Bibi, mais à une linotte : "Voilà une linotte bien galante ", affirme Abor. Loin de se présenter comme une allégorie, le conte relance le processus herméneutique, et emporte le lecteur dans le déchiffrement des signes. Le lecteur suit ainsi lui-même un mouvement initiatique. Lorsqu'au cours de la lecture, la mise en réseau des nombreux symboles et les effets d'analogies apparaissent plus nettement, lorsque l'hermétisme apparent du texte s'estompe, la joie du lecteur est à la fois liée au plaisir intellectuel du déchiffrage de l'énigme, mais également à l'interrogation morale et philosophique que le texte suscite. C'est en ce sens que le conte philosophique a une fonction éthique.

72 Ibid., p. 190.

73 Thémiseul de Saint-Hyacinthe, t. 27, 1. III, op. cit., p. 487.

74 Martha Nussbaum, "La littérature comme philosophie morale. La fêlure dans le cristal : La coupe d'or de Henri James ", dans Éthique, littérature, vie humaine, éd. Sandra Laugier, Paris, PUF, 2006, p. 42.

75 Ibid., t. 28, 1. IV, p. 49. 
En conclusion, si ces trois auteurs de contes philosophiques semblent bien loin de former une quelconque communauté de pensée, la forte dimension symbolique de leurs textes permet au lecteur de se mettre à la place des personnages, non pas par un processus d'identification, comme dans le roman épistolaire, mais par un procédé de mise en abyme et de distanciation : grâce au dispositif narratif, le conte met en scène sa propre réception. Le double mouvement ainsi créé, de plongée dans la fiction et de mise à distance, permet l'éveil des consciences : le sujet émerge grâce au détour par l'Autre, mais surtout dans la prise de conscience de l'analogie entre le singulier et l'universel. Si un " espace public » se dessine, au sens d' " espace dans lequel les individus autonomes peuvent se coordonner ${ }^{76}$ ", il est seulement au cœur du texte ; mais c'est ce qui confere sans doute à la littérature, comme expérience de l'altérité, une force politique et éthique. En ce sens, la littérature et les arts apparaissent comme des fondements de la démocratie, comme l'a montré Martha Nussbaum, dans son ouvrage intitulé Les émotions démocratiques ${ }^{77}$. Le conte philosophique participe ainsi au développement d'une communauté de lecteurs engagés dans une même quête du savoir et dans un même processus de décryptage du sens, ce qui définit le lien social par excellence au XVIII siècle, comme l'affirme d'Alembert dans le Discours préliminaire de l'Encyclopédie : «Les premiers hommes, en s'aidant mutuellement de leurs lumières, c'est-à-dire de leurs efforts séparés ou unis, sont parvenus, en assez peu de temps, à découvrir une partie des usages auxquels ils pouvaient employer les corps ${ }^{78}$. "L'étude de ces quatre contes philosophiques a montré que cette "sociabilité des Lumières ", s'exprime non seulement dans les essais, dans les assemblées, mais également au cœur même de la fiction qui émerge dans la première moitié du XVIII siècle, ce qui fait dire à David J. Denby, que «la naissance du social est esthétique ${ }^{79}$ ».

Magali Fourgnaud

EA $4574 \mathrm{SPH}$

Université Michel de Montaigne Bordeaux 3

Magali.fourgnaud@orange.fr

76 David J. Denby, art. cit., p. 137.

77 Martha Nussbaum, Les émotions démocratiques, comment former le citoyen du XXI' siècle ?, titre original : Not for profit. Why démocracy Nedds the Humanities, Paris, Climats-Flammarion, 2011.

78 D'Alembert, Discours préliminaire de l'Encyclopédie, cité par Catherine Larrère, art. cit., p. 11491150.

79 David J. Denby, art. cit., p. 131. 


\title{
Résumé
}

Dans la première moitié du XVIII ${ }^{e}$ siècle, le conte à visée morale et philosophique témoigne de l'émergence de nouveaux modèles de sociabilité. D'une part, il met en scène des sociétés unies par la quête de la vérité et du bonheur individuel et collectif. D'autre part, le dispositif narratif permet au lecteur de prendre littéralement la place de l'Autre : esthétique sentimentale et développement de l'idée de démocratie se trouvent ainsi intimement liées.

\section{Mots-clés}

Conte philosophique, sociabilité, esthétique sentimentale, récit initiatique.

\begin{abstract}
In the first half of the eighteenth century, the development of the philosophical tale corresponds to the emergence of new forms of sociability. On the one hand, it depicts societies which share the quest for truth as well as for individual and collective happiness. On the other hand, the narrative devices allow the reader to substitute himself to "the Other": the sentimentalist aesthetics and the development of the idea of democracy are thus closely related.
\end{abstract}

\section{Keywords}

Philosophical tale, sociability, sentimentalist aesthetics, initiation story. 